\title{
Research strategies and the use of nutrient biomarkers in studies of diet and chronic disease
}

\author{
Ross L Prentice ${ }^{1,2, *}$, Elizabeth Sugar ${ }^{1,3}$, CY Wang ${ }^{1,2}$, Marian Neuhouser ${ }^{1}$ and \\ Ruth Patterson 1,4 \\ 'Division of Public Health Sciences, Fred Hutchinson Cancer Research Center, 1100 Fairview Avenue North, \\ Seattle, WA 98109, USA: ${ }^{2}$ Department of Biostatistics, University of Washington, Seattle, WA, USA: \\ ${ }^{3}$ Department of Statistics, University of Washington, Seattle, WA, USA: ${ }^{4}$ Department of Epidemiology, \\ University of Washington, Seattle, WA, USA
}

\begin{abstract}
Objective: To provide an account of the state of diet and chronic disease research designs and methods; to discuss the role and potential of aggregate and analytical observational studies and randomised controlled intervention trials; and to propose strategies for strengthening each type of study, with particular emphasis on the use of nutrient biomarkers in cohort study settings.

Design: Observations from diet and disease studies conducted over the past 25 years are used to identify the strengths and weaknesses of various study designs that have been used to associate nutrient consumption with chronic disease risk. It is argued that a varied research programme, employing multiple study designs, is needed in response to the widely different biases and constraints that attend aggregate and analytical epidemiological studies and controlled intervention trials. Study design modifications are considered that may be able to enhance the reliability of aggregate and analytical nutritional epidemiological studies. Specifically, the potential of nutrient biomarker measurements that provide an objective assessment of nutrient consumption to enhance analytical study reliability is emphasised. A statistical model for combining nutrient biomarker data with self-report nutrient consumption estimates is described, and related ongoing work on odds ratio parameter estimation is outlined briefly. Finally, a recently completed nutritional biomarker study among 102 postmenopausal women in Seattle is mentioned. The statistical model will be applied to biomarker data on energy expenditure, urinary nitrogen, selected blood fatty acid measurements and various blood micronutrient concentrations, and food frequency self-report data, to identify study subject characteristics, such as body mass, age or socio-economic status, that may be associated with the measurement properties of food frequency nutrient consumption estimates. This information will be crucial for the design of a potential larger nutrient biomarker study within the cohort study component of the Women's Health Initiative.

Setting and subjects: The methodology under study is expected to be pertinent to a wide variety of diet and chronic disease association studies in the general population. Ongoing work focuses on statistical methods developed using computer simulations motivated by studies of dietary fat in relation to breast and colon cancer among postmenopausal women, and ongoing pilot studies to be described in detail elsewhere, involving post-menopausal women living in the Seattle area.

Results and conclusion: A varied research programme appears to be needed to make progress in the challenging diet and chronic disease research area. Such progress may include aggregate studies of diet and chronic disease that include sample surveys in diverse population groups world-wide, analytical epidemiological studies that use nutrient biomarker data to calibrate self-report nutrient consumption estimates, and randomised controlled intervention trials that arise from an enhanced infrastructure for intervention development. New innovative designs, models and methodologies are needed for each such research setting.
\end{abstract}

Keywords

Chronic disease

Dietary measurement error Nutrient consumption biomarkers Odds ratio Systematic bias Research strategies
The relationships between nutrient consumption and chronic disease risk have been the focus of a large number of epidemiological studies for the past three decades. While these studies have identified many new leads ${ }^{1}$, there has also been much controversy ${ }^{2,3}$ with less consistency than one might expect among the reports from ecological, case-control and cohort studies. As a result, few causal diet and chronic disease associations can be said to be well 
established. Important exceptions include the relationship between the components of dietary fat and coronary heart disease and to a lesser extent the relationship between calcium consumption and fracture risk. In both cases a biomarker was identified that was responsive to dietary consumption - namely, plasma lipoproteins and bone mineral density, respectively - and interventions that favourably affect the biomarker were developed and subsequently shown to reduce the risk of the related chronic disease in question in randomised controlled trials ${ }^{4-6}$. It is interesting to note that, in these contexts, observational studies to relate self-reported fat intake to coronary heart disease, and observational studies to relate calcium consumption to fracture incidence, have tended to be unimpressive in their clarity or consistency.

Analytical epidemiological studies in the diet and chronic disease area face formidable challenges. First, there are the usual ascertainment and confounding bias issues. For example, persons who consume a healthful diet may also tend to have atypical health-related habits in such areas as physical activity patterns, avoidance of tobacco products and environmental exposures, and disease screening. In addition, there are often key limitations related to the nutrient consumptions themselves. Within study populations there may be limited variation in nutrient consumption; for example, most persons in the USA currently consume a diet having between 25 and 35\% energy from fat. Also, the diet is a complex mixture of foods and nutrients with many correlated elements. Hence one may need to be able to measure such components with exquisite precision on individuals to distinguish the roles of such foods and nutrients in analytical epidemiological studies.

Unfortunately, available dietary self-assessment tools do not provide such precision, because related consumption estimates are known to involve substantial random error for most nutrients, and probably incorporate important systematic error as well. Available statistical tools do not make provision for such systematic errors, casting an important shadow over virtually all analytical epidemiological studies of diet and chronic disease that have been reported to date.

Per capita food disappearance data ${ }^{7}$, deriving from food production with adjustments for imports and exports and for food consumption by animals, have been a source of dietary data for many nations world-wide for a sustained period of time. These data are crude; for example, they are not even age- or sex-specific. However, they have the advantage of objectivity in that self-reporting is not involved. Some authors have reported strong associations of per capita disappearance of selected nutrients with disease incidence and mortality rates ${ }^{8-10}$, especially for cancer, where good-quality population-based disease registers exist in many countries. Unfortunately, to date, these ecological studies have lacked the ability to make adequate provision for between-population confounding.
This potential confounding, along with limitations of the nutrient supply (disappearance) data, has caused these studies to be relegated to a hypothesis-generating role.

Randomised controlled trials in the area of diet and chronic disease have the potential to overcome many of the limitations of the ecological and analytical epidemiological studies. First, randomisation implies a lack of confounding of the intervention comparison with prerandomisation dietary and non-dietary factors. Second, individual dietary assessment no longer constitutes the critical exposure data at the core of the study design, as with analytical epidemiological studies, but rather addresses the secondary questions about the assessment of adherence to intervention goals and about the explanation of disease rate differences between intervention and control groups. However, randomised trials are expensive and logistically difficult, and may need to continue for a lengthy period of time to provide an adequate assessment of the health benefits versus risks for the interventions under test. As a result, very few individually randomised dietary intervention trials have been conducted and most completed ongoing trials have involved pill-taking interventions. Intervention trials that involve alterations of dietary patterns and strong behavioural components are a more recent phenomenon. These trials would seem to be of very high priority although they will often have the additional complication that intervention and control groups differ in various aspects of dietary and non-dietary behaviour, beyond the intended dietary differences.

The above description highlights the genuine difficulties in generating reliable information in the diet and chronic disease research area. The next section argues the need for a varied research programme that strengthens the execution of each of the study designs mentioned above.

\section{Aspects of a comprehensive diet and chronic disease research programme}

Consider a cohort study that relates nutrient intakes, as assessed using food frequencies, food records or food recalls, to the subsequent occurrence of a certain disease. Sometimes the intake assessments for a particular nutrient are claimed to be 'validated' merely on the basis of a positive correlation between the assessments on individual study subjects using different self-report instruments, or a positive correlation between repeat applications of the same instrument. However, the measurement errors associated with multiple assessments on a particular study subject are very likely also to be positively correlated, making it unclear from readily available data whether the instruments used in the multiple assessments are in any sense measuring the nutrient exposure of interest. For example, if obese persons systematically underreport energy consumption compared with lean persons, then, regardless of the assessment instrument used, energy 
consumption measurement errors will tend to be positively correlated across assessment instruments, and what appears as a consistency in nutrient assessment may be attributable, in whole or in part, to a consistency in misreporting. In fact, studies using doubly labelled water assessments of short-term energy expenditure ${ }^{11}$ suggest strongly that obese persons, in Western populations, do systematically underreport energy consumption, as is also the case, but to a lesser extent, for the self-reporting of protein consumption based on studies using urinary nitrogen evaluations ${ }^{11}$. Of course obesity, or more generally body mass, is but one of the study subject characteristics that may be associated with the systematic bias in dietary self-reporting. Other such factors may include gender, age, ethnicity, socio-economic status and social desirability factors ${ }^{12,13}$, to name a few.

Available statistical models for accommodating exposure measurement error almost universally require the availability of two (or more) exposure assessments having uncorrelated measurement errors. As noted above, an uncorrelated measurement error assumption for nutrient intake estimates based on different self-report instruments, or on repeated applications of a single selfreport instrument, may be quite implausible. On the other hand, assuming uncorrelated measurement errors between the nutrient consumption estimate arising from a particular self-report instrument and that derived from a suitable objective biomarker of nutrient assumption may be much more plausible. Furthermore, a simple classical measurement model may apply to the nutrient biomarker assessment, making it a sensible candidate to calibrate a corresponding self-report assessment, which will typically be the only nutrient assessment available on most members of the study cohort. The actual calibration equations will depend on the nature of the systematic and random error elements of the self-report nutrient consumption estimates. In the next section we describe a statistical model for bringing together biomarker nutrient consumption data, on a moderate sized subset of a study cohort, with self-report nutrient assessment data on the entire cohort, to relate an underlying true consumption to disease risk. This is followed by a brief overview of a nutrient biomarker pilot study that we have conducted among 102 postmenopausal women in Seattle.

Before going into these topics in more detail, we shall make a few comments on the role that population comparisons and randomised controlled intervention trials can play in the diet and chronic disease research agenda, and outline some ways of strengthening these studies.

Students of epidemiology are typically taught a hierarchy of increasing study reliability starting from ecological studies, and ranging through case-control studies, cohort studies and intervention trials. However, in circumstances of difficult-to-measure, highly correlated exposures and difficult-to-measure confounding factors, such a hierarchy may not always hold. In particular, well-conducted ecological studies, in which moderate sized random samples in each group of a multi-population aggregate study are surveyed to ascertain exposures and potential confounding factors, are largely immune to the noise aspect of measurement error ${ }^{14,15}$. Such studies may be less sensitive than cohort or case-control studies to systematic aspects of measurement error as well, in part due to their ability to incorporate an unusually broad range of exposures. It is not clear how successfully 'between-population' confounding can be controlled in such settings, but the challenges and uncertainties of observational studies in the diet and disease area argue for a varied research programme, including research designs having differing sources of potential bias. Furthermore, methodological work towards defining the comparative reliability of various observational approaches as a function of the distribution and measurement properties of key exposure and confounding variables should have a high priority in the chronic disease population research agenda.

Our group in Seattle has carried out substantial planning activities for a potential international study on aggregate data of diet and cancer. The study would be conducted in about 35 diverse populations world-wide that are covered by good-quality cancer registries. In each population, 300 males and 300 females would be randomly selected from persons aged 35-64 years in the registry catchment area, and epidemiological questionnaire data and blood specimens would be obtained. Regression analyses would relate disease risk to the distribution of dietary exposure and confounding factor data, with biomarker data providing the possibility of avoiding the need to rely on difficult-to-standardise dietary self-assessment tools. This study design has been piloted in Shanghai, Costa Rica and Seattle ${ }^{16}$.

Diet and chronic disease prevention hypotheses having substantial public health potential should be put to test in randomised controlled intervention trials whenever practical. Recent examples include colon polyp prevention trials using calcium supplementation ${ }^{17}$, wheat bran fibre supplementation ${ }^{18}$ and a low-fat eating pattern ${ }^{19}$, and the ongoing Women's Health Initiative (WHI) ${ }^{20}$ clinical trial of a low-fat eating pattern among 48836 postmenopausal women in the United States for the prevention of breast and colorectal cancer and, secondarily, coronary heart disease. As mentioned previously, however, randomised controlled trials of dietary interventions having disease outcomes are logistically difficult and expensive. Hence there is a pressing need for a strong intervention development enterprise, and careful feasibility and pilot studies are needed prior to embarking on such trials.

In addition to various types of observational study, an adequate intervention development enterprise is likely to rely heavily on small-scale human feeding studies with intermediate biochemical, and perhaps genomic or 
proteomic, outcomes. In particular, the rapid development of functional genomics ${ }^{21}$ using micro-array technologies, and the potential of high-dimensional protein array data in the not-too-distant future, may add much specificity to the screening of preventive interventions. These technologies may allow one to observe the simultaneous effect of an intervention on the expression of a large number of human genes, or expressed sequence tags, throughout the human genome, or a large number of protein concentrations, in order to provide insight into a broad range of potential intervention effects. The statistical and bioinformatic aspects of analysing and interpreting such highdimensional data are at an early stage of development, but are widely recognised as critical to the impact of genomic techniques. Such data-rich techniques also enter the chronic disease population research area in the identification of persons at high risk for specific diseases, which may be able to lead to smaller and more efficient prevention trials, and to aid in the elucidation of geneenvironment interactions that may have a substantial role in chronic disease development.

\section{Statistical methods to combine nutrient biomarker data with self-report nutrient consumption data in epidemiological cohort studies}

Consider a nutrient consumption value $Z$ for an individual. For example, $Z$ may be the individual's long-term daily average energy consumption, or a corresponding daily average nutrient density. An objective biomarker measure $W$, of $Z$, may plausibly adhere to a classical measurement model

$$
W=Z+u,
$$

where $u$ is a mean zero error variable that is independent of $Z$ and independent of other study subject characteristics. Such a biomarker measurement provides the 'anchor' that allows self-report assessments $Q$ of the same nutrient consumption to be calibrated. Because it is usually impractical to obtain biomarker data on the entire study cohort, which may be tens of thousands in size, selfreport estimates of nutrient consumption will need to be used as the sole source of dietary data on most study subjects. Also, because the measurement error associated with estimates of $Z$ may depend on a vector $V$ of study subject characteristics (e.g. body mass, age, social desirability factors, etc.), one might consider a statistical model of the form

$$
Q=Z^{*}+\varepsilon
$$

where $\varepsilon$ is a random error term independent of $Z^{*}$ and all study subject characteristics and $Z^{*}$ is the actual 'target' of the self-report assessment that could plausibly be expressed as

$$
Z^{*}=\gamma_{0}+\gamma_{1} Z+\gamma_{2}^{T} V+\gamma_{3}^{T} V Z+\eta
$$

where $\eta$ is a person-specific random effect and ' $T$ ' denotes vector transpose. Note that the term $\gamma_{3} V Z$ makes a provision for systematic bias in that, if a component of $\gamma_{3}$ is non-zero, then there is systematic bias in the self-report assessment of $Z$ that is associated with the corresponding element of $V$. Also, the mean zero random effect, $\eta$, accommodates a further person-specific bias. Its variance is allowed to depend on $V$, but when re-scaled to have unit variance the random effect is assumed to be independent of $(V, Z)$. The random effect allows the measurement errors in repeat self-report assessments of $Z$ to be correlated. Related measurement models for $Q$ have previously been considered ${ }^{22,23}$. Note that repeat application of the biomarker measurement $W$ and the selfreport measurement $Q$, with independent measurement errors in (1) and (2) respectively, are needed on subsamples of study subjects to estimate the variances of $u$ and $\varepsilon$. Key requirements in the use of the measurement model (1)-(3) include the availability of an objective measure $(W)$ to which a classical measurement model plausibly applies and the accommodation, by means of $V$, of the characteristics that are associated with systematic bias in the self-report assessment $W$. Under these circumstances, one can use nutrient consumption estimates $Q$ on the cohort and biomarker estimates $W$ on an appropriate subset of the cohort to relate the underlying nutrient consumption $Z$ to disease occurrence in the cohort. For example, one may have a binary disease indicator $(Y)$ and consider a logistic regression model

$$
\operatorname{Pr}(Y=1 \mid Z, V)=\left\{1+\exp \left[-\left(\beta_{0}+\beta_{1} Z+\beta_{2}^{T} V\right)\right]\right\}^{-1} .
$$

The parameter $\beta_{1}$ that relates the odds of disease to the true nutrient exposure $Z$ would then be of primary interest in data analysis.

It is of interest to consider the bias in estimators of $\beta_{0}, \beta_{1}$ and $\beta_{2}$ that may arise under standard methods of data analysis that fail to account fully for the elements of the measurement model (1)-(3). To do so, we generated normally distributed exposures $Z$ having mean and variance both equal to one, and obtained corresponding pairs of $W$ (biomarker) values by adding to $Z$ independent error variates that were normally distributed with a mean of zero and a variance of one. Corresponding self-report 'targets' $Z^{*}$ were generated using (3) with $\gamma_{0}=0.0, \gamma_{1}=$ $0.8, \gamma_{2}=0.2$ and $\gamma_{3}=0.2$, with $V$ a univariate characteristic (e.g. body mass), independent of $Z$, that takes values $-1,0$ and 1 with respective probabilities $0.2,0.6$ and 0.2 , and with $\eta /(1+0.5 V)^{1 / 2}$ as standard normal variate, independent of $(V, Z)$. Paired self-report $Q$ values were generated according to (2) by adding to $Z^{*}$ normal error variates having mean zero and variance 0.5 that are independent of $Z, V$ and $\eta$. Binary disease indicators ( $Y=1$ for diseased; $Y=0$ otherwise) were generated from $Z$ and $V$ using (4) with $\beta_{0}=-\log 2=-0.6931$, $\beta_{1}=\log 2=0.6931$ and $\beta_{2}=0$. Five hundred replicates 
of cohort datasets, each of size 100 and 500, were generated and various potential estimators of $\beta_{0}$ and $\beta_{1}$ were calculated ( $\beta_{2}$ was set to zero throughout).

Table 1 provides simulation summary statistics for four estimators of $\beta_{0}$ and $\beta_{1}$. The column labelled $\bar{Q}$ is based on binary logistic regression of $Y$ with the average $(\bar{Q})$ of the corresponding self-report assessments of $Z$ in place of the actual $Z$ values. Very substantial biases in $\beta_{0}$ and $\beta_{1}$ estimators are evident and confidence interval coverage probabilities are not close to their nominal value of 0.95 . A second estimator replaces $Z$ in the logistic regression (4) by $\bar{W}$, the average of the paired biomarker values. Biases remain large and confidence interval coverage rates are still poor. A third, less naïve, estimator would replace $Z$ in (4) by an estimator $E(Z \mid Q)$ of the expectation of the true exposure $Z$ given the pair of corresponding $Q$ values, assuming that these self-report values arise from a classical measurement model $Q=Z+\varepsilon$, rather than (3). This is the regression calibration method ${ }^{24}$ that may be used if the self-report data are assumed to be without systematic bias or, equivalently, if multiple self-report assessments are assumed to have statistically independent measurement errors. Table 1 shows the resulting estimators of relative risk parameters to be seriously biased, with the $\beta_{1}$ estimate strongly attenuated towards zero. Finally, Table 1 shows an appropriate regression calibration estimator in which $Z$ in (4) is replaced by $E(Z \mid W)$, an estimate of the expectation of $Z$ given the pair of biomarker $W$ values. This estimate was based on simple linear calibration equations, and a corresponding 'sandwich-type' variance estimator was calculated for the $\left(\beta_{0}, \beta_{1}\right)$ estimates. As shown in Table 1 , these variance estimates align closely with the corresponding sample variances and confidence intervals have close-to-nominal coverages. In addition, the biases in the estimators of $\left(\beta_{0}, \beta_{1}\right)$ appear to be negligible.

In the simulation study just described, we assumed $W$ (biomarker) determinations to be available on the entire study cohort. As noted above, this will not be practical in a nutritional epidemiology cohort study. We have conducted additional development of calibration estimators of regression parameters, which involve replacing $Z$ in (4) by an estimate of its expectation conditional on $V$ and on the $W$ and $Q$ values that are available for the study subject in question. We have also conducted corresponding simulation studies that show that such methods can lead to odds ratio parameter estimates having acceptable bias and coverage properties even if biomarker data are available on only a modest fraction of the study cohort. Our ongoing work, to be described in detail elsewhere, is also looking into the necessary size of a biomarker sub-study as a function of the complexity of the measurement properties of $Q$, as reflected in (2) and (3). This work, along with biomarker pilot data to build a measurement model (1)-(3), is intended to provide input to the design of a potential future nutrition biomarker sub-study of the WHI cohort study.

There appears to have been little discussion in the literature as to the types of biomarker that may adhere to a classical measurement model (1). These measures, such as doubly labelled water assessments of energy expenditure

Table 1 Simulation study of various estimators of logistic regression parameters $\beta_{0}=-0.6931$ and $\beta_{1}=0.6931$ with biomarker estimates $W$, and self-report estimates $Q$, of a nutrient exposure $Z$ generated according to the measurement model (1)-(3). The estimates labelled $\bar{Q}$ and $\bar{W}$ are obtained by replacing $Z$ values by the average of paired $Q$ and $W$ values in logistic regression, while those labelled $E(Z \mid Q)$ and $E(Z \mid W)$ are regression calibration estimates obtained under an oversimplified and correct measurement model assumption, respectively

\begin{tabular}{|c|c|c|c|c|c|c|}
\hline \multirow[b]{2}{*}{ Cohort size } & \multirow[b]{2}{*}{ Parameter } & & \multicolumn{4}{|c|}{$\begin{array}{l}\text { Regression } \\
\text { variable } \\
(Z) \text { replacements }\end{array}$} \\
\hline & & & $\bar{Q}$ & $\bar{W}$ & $E(Z \mid Q)$ & $E(Z \mid W)$ \\
\hline \multirow[t]{8}{*}{100} & \multirow{4}{*}{$\beta_{0}$} & Sample bias & 0.454 & 0.225 & 0.436 & -0.024 \\
\hline & & Sample SD* & 0.246 & 0.291 & 0.252 & 0.373 \\
\hline & & Average SD estimate* & 0.246 & 0.282 & 0.251 & 0.360 \\
\hline & & $95 \% \mathrm{Cl}^{*}$ & 0.520 & 0.850 & 0.554 & 0.952 \\
\hline & \multirow{4}{*}{$\beta_{1}$} & Sample bias & -0.404 & -0.226 & -0.382 & 0.023 \\
\hline & & Sample SD* & 0.169 & 0.191 & 0.182 & 0.301 \\
\hline & & Average SD estimate* & 0.160 & 0.188 & 0.172 & 0.290 \\
\hline & & $95 \% \mathrm{Cl}^{*}$ & 0.280 & 0.728 & 0.384 & 0.958 \\
\hline \multirow[t]{8}{*}{500} & \multirow[t]{4}{*}{$\beta_{0}$} & Sample bias & 0.463 & 0.247 & 0.446 & 0.018 \\
\hline & & Sample SD* & 0.112 & 0.126 & 0.114 & 0.161 \\
\hline & & Average SD estimate* & 0.108 & 0.123 & 0.110 & 0.154 \\
\hline & & $95 \% \mathrm{Cl}^{*}$ & 0.102 & 0.486 & 0.022 & 0.934 \\
\hline & \multirow[t]{4}{*}{$\beta_{1}$} & Sample bias & -0.418 & -0.246 & -0.398 & -0.017 \\
\hline & & Sample SD* & 0.070 & 0.079 & 0.075 & 0.125 \\
\hline & & Average SD estimate* & 0.069 & 0.081 & 0.074 & 0.123 \\
\hline & & $95 \% \mathrm{Cl}^{*}$ & 0.000 & 0.154 & 0.006 & 0.946 \\
\hline
\end{tabular}

* The entries are the sample standard deviation, the average of standard deviation estimates and the empirical coverage rate of nominal $95 \%$ confidence intervals. 
and urinary nitrogen assessments of protein expenditures, which involve collecting a metabolite that arises if and only if the corresponding nutrient is expended, the abundance of which reflects the corresponding amount of nutrient expended, are likely to be the most valuable. These measures still require the metabolite collection to be complete, and the study subject to be in nutrient balance, and they require calibration equations that express consumption estimates as a function of metabolite measurements.

Some other potential biomarkers, for example blood concentrations of certain micronutrients, may typically be influenced by factors beyond recent consumption of the nutrient. Such biomarkers will then not adhere to the simple measurement model (1) and may themselves require a substantial calibration study to develop a suitable measurement model. Even so, a biomarker in (1) would, however, have a major advantage over a second selfreport assessment (e.g. using food records) in that the biomarker measurement error $(u)$ may plausibly be taken to be uncorrelated with that $(\varepsilon)$ for the self-report estimate, whereas it would seem virtually impossible to argue convincingly that two self-report assessments have uncorrelated measurement errors, regardless of the complexity of models (1) and (2). Even with a biomarker in (1) one should be aware of the possibility of positively correlated errors in (1) and (2), and hence of optimistic assessment of the properties of the measurement $Q$, simply because both assessments may aim to measure consumption in a common narrow time window (e.g. a few weeks or months) while the target $Z$ may be defined to reflect consumption over a much longer time period (e.g. a decade or two). Much important work remains to be carried out on the selection, modelling and use of appropriate biomarkers of nutrient (and food group) consumption.

\section{WHI nutrition biomarker pilot study}

As discussed above, error in self-report of dietary intake is perhaps the single greatest impediment to understanding the effect of diet on disease risk. However, for many studies, self-report (often food-frequency questionnaires (FFQs)) will be the only dietary assessment on all participants because of logistic and cost constraints. Therefore it has become increasingly clear that largescale research efforts need to incorporate biomarker sub-studies that can identify and quantify sources of measurement error in the parent study's primary dietary assessment instrument.

The WHI nutrition biomarker pilot study was designed to demonstrate the feasibility of collecting a comprehensive panel of biological markers of dietary intake for comparison with the WHI FFQ ${ }^{25}$. The WHI is perhaps the most ambitious population research investigation ever undertaken. Briefly, this initiative includes a randomised, controlled clinical trial to evaluate the health benefits and risks of three distinct interventions among 68133 postmenopausal women and an observational study comprised of 93676 postmenopausal women. Follow-up of participating women is planned through March 2005 for an average of about 8.5 years' duration $^{20}$. The potential for this observational study to make important new contributions to our understanding of diet and disease will be influenced heavily by the degree to which we can correct for systematic and personspecific measurement error in the FFQ. Below we provide an overview of the protocol employed in the WHI nutrition biomarker pilot study, which was designed to address issues of error in dietary self-report.

This pilot study obtained dietary intake data using the WHI FFQ along with objective measures of intake from 102 healthy postmenopausal women aged 50-79 years in Seattle, Washington. The biomarker data include measures of energy (using indirect calorimetry and an accelerometer), protein (from urinary nitrogen), carbohydrate (using a phospholipid fatty acid), and vitamin C, folate, retinol, tocopherols and carotenoids (from fasting blood samples).

We recruited participants with the same methods used to recruit women for WHI: mass mailings and public service announcements. Women attended two clinic visits (one week apart) so that we could obtain duplicate measures of key variables for purposes of investigating reliability and within- vs. between-person variability. At each visit we measured resting energy expenditure using indirect calorimetry, took a fasting blood sample, and obtained a 24-hour urine sample that women collected the day preceding the clinic visit. For measurement of activityrelated energy expenditure, participants wore an accelerometer during waking hours for at least two nonconsecutive days between clinic visits. We also measured height, weight and body circumferences. In addition to the FFQ, participants completed questionnaires on demographic characteristics, health behaviour and psychosocial factors such as social desirability. Only two women began the protocol and did not finish the study (98\% completion rate). Participants were paid $\$ 100$ as compensation for their time and effort.

For the biomarker of energy intake, we encountered an unanticipated problem because of the market unavailability of doubly labelled water for determination of energy expenditure ${ }^{26}$. Therefore we developed an alternative protocol for measuring the major components of energy needs: (1) resting metabolic rate determined using an indirect calorimeter (SensorMedics, Yorba Linda, CA) and (2) activity-related energy expenditure using a Caltrac accelerometer (Muscle Dynamics, Torrance, CA). Some properties of this energy expenditure measure have recently been reported ${ }^{27}$.

For the biomarker of protein intake, we obtained two 
24-hour urine collections. The use of urinary nitrogen as a marker of protein intake is well studied and controlled feeding studies have shown that, over a 28-day period of time, 24-hour urinary nitrogen correlates nearly perfectly with dietary nitrogen intake, representing $78-83 \%$ of intake ${ }^{28}$.

A potential biomarker of carbohydrate intake is a plasma phospholipid fatty acid called vaccenic acid $(18: 1 n-7)$ that is produced endogenously from carbohydrate and appears to increase in response to a low-fat diet. This biomarker was identified using a controlled feeding study comparing fasting plasma fatty acid profiles among postmenopausal women on a low-fat (highcarbohydrate) diet vs. a high-fat (low-carbohydrate) diet. Results of this study have provided preliminary evidence that plasma phospholipid fatty acids may be able to serve as quantitative biomarkers of total dietary fat and/or carbohydrate intake ${ }^{29}$. For example, using discriminant analyses, phospholipid vaccenic acid concentrations distinguished between the two controlled diets almost perfectly. Finally, we propose to obtain an objective measure of fat (and alcohol) intake by subtracting protein and carbohydrate intakes (determined from the biomarkers) from our measure of energy intake.

Further analyses from this pilot study are currently underway and may be used to inform the design of a larger nutritional biomarker study within the WHI Observational Study. For this purpose, the pilot study data can provide preliminary estimates of the calibration function (3) for the food frequency nutrient assessments by identifying factors ( $V$ in equation (3)) that are related to the measurement error properties of the FFQ and by providing estimates of the corresponding calibration parameters $(\gamma)$.

\section{Acknowledgements}

This work was supported by NIH programme project grant CA53996 and by a grant from the Fannie E. Rippel Foundation, Basking Ridge, NJ.

\section{References}

1 World Cancer Research Fund (WCRF)/American Institute for Cancer Research (AICR). Food, Nutrition and the Prevention of Cancer: A Global Perspective. Washington, DC: WCRF/AICR, 1997.

2 Greenwald P. Role of dietary fat in the causation of breast cancer: point. Cancer Epidemiol. Biomark. Prev. 1999; 8: 3-7.

3 Hunter DJ. Role of dietary fat in the causation of breast cancer: counterpoint. Cancer Epidemiol. Biomark. Prev. 1999; 8: 9-13.

4 Shepherd J, Cobbe SM, Ford I, Isles CG, Lorimer AR, Macfarlane PW, et al. Prevention of coronary heart disease with pravastatin in men with hypercholesterolemia. West of Scotland Coronary Prevention Study Group. N. Engl.J. Med. 1995; 333: 1301-7.

5 Chapuy MC, Arlot ME, Duboeuf F, Brun J, Crouzet B, Arnaud $\mathrm{S}$, et al. Vitamin $\mathrm{D}_{3}$ and calcium to prevent hip fractures in the elderly women. N. Engl. J. Med. 1992; 327: 1637-42.
6 Cummings SR, Black DM, Thompson DE, Applegate WB, Barrett-Connor E, Musliner TA, et al. Effect of alendronate on risk of fracture in women with low bone density but without vertebral fractures - results from the fracture intervention trial. Fracture Intervention Trial Research Group. J. Am. Med. Assoc. 1998; 280: 2077-82.

7 Food and Agriculture Organization (FAO). Food Balance Sheets, 1975-1977 Average: and Per Caput Food Supplies, 1961-65 Average, 1967 to 1977. Rome: FAO, 1980.

8 Armstrong B, Doll R. Environmental factors and cancer incidence and mortality in different countries, with special reference to dietary practices. Int. J. Cancer 1975; 15: 617-31.

9 Gray GE, Pike MC, Henderson BE. Breast-cancer incidence and mortality rates in different countries in relation to known risk factors and dietary practices. Br.J. Cancer 1979; 39: 1-7.

10 Prentice RL, Sheppard L. Dietary fat and cancer: consistency of the epidemiologic data, and disease prevention that may follow from a practical reduction in fat consumption. Cancer Causes Control 1990; 1: 81-97.

11 Heitmann BL, Lissner L. Dietary underreporting by obese individuals: is it specific or non-specific? Br. Med. J. 1995; 311: 986-9.

12 Hebert JR, Clemow L, Pbert L, Ockene IS, Ockene JK. Social desirability bias in dietary self-report may compromise the validity of dietary intake measures. Int. J. Epidemiol. 1995; 24: 389-98.

13 Hebert JR, Ma Y, Clemow L, Ockene IS, Saperia G, Stanek EJ III, et al. Gender differences in social desirability and social approval bias in dietary self-report. Am. J. Epidemiol. 1997; 146: $1046-55$.

14 Prentice RL, Sheppard L. Aggregate data studies of disease risk factors. Biometrika 1995; 82: 113-25.

15 Sheppard L, Prentice RL. On the reliability and precision of within- and between-population estimates of relative risk parameters. Biometrics 1995; 51: 853-63.

16 Satia JA, Patterson RE, Herrero R, Jin F, Dai Q, King IB, et al. Study of diet, biomarkers and cancer risk in the United States, China and Costa Rica. Int. J. Cancer 1999; 82: 28-32.

17 Baron JA, Beach M, Mandel JS, van Stolk RU, Haile RW, Sandler RS, et al. Calcium supplements for the prevention of colorectal adenomas. Calcium Polyp Prevention Study Group. N. Engl. J. Med. 1999; 340: 101-7.

18 Alberts DS, Martínez ME, Roe DJ, Guillén-Rodríguez JM, Marshall JR, Van Leeuwen JB, et al. The Phoenix Colon Cancer Prevention Physician's Network. Lack of effect of a high-fiber cereal supplement on the recurrence of colorectal adenomas. N. Engl. J. Med. 2000; 342: 1156-62.

19 Schatzkin A, Lanza E, Corle D, Lance P, Iber F, Caan B, Shike $\mathrm{M}$, et al. The Polyp Prevention Trial Study Group. Lack of effect of a low-fat, high-fiber diet on the recurrence of colorectal adenomas. N. Engl. J. Med. 2000; 342: 1149-55.

20 The Women's Health Initiative Study Group. Design of the Women's Health Initiative clinical trial and observational study. Control. Clin. Trials 1998; 19: 61-109.

21 Brown PO, Hartwell L. Genomics and human disease variations on variation. Nat. Genet. 1998; 18: 91-3.

22 Prentice RL. Measurement error and results from analytic epidemiology: dietary fat and breast cancer. J. Natl. Cancer Inst. 1996; 88: 1738-47.

23 Kipnis V, Carroll RJ, Freedman LS, Li L. Implications of a new dietary measurement error model for estimation of relative risk: application to four calibration studies. Am.J. Epidemiol. 1999; 150: 642-51.

24 Carroll RJ, Ruppert D, Stefanski LA. Measurement Error in Nonlinear Models. London: Chapman \& Hall, 1995.

25 Patterson RE, Kristal AR, Fels-Tinker L, Carter RA, Bolton MP, Agurs-Collins T. Measurement characteristics of the Women's Health Initiative Food Frequency Questionnaire. Ann. Epidemiol. 1999; 9: 178-97. 
26 Schoeller DA. Recent advances from application of doubly labeled water to measurement of human energy expenditure. J. Nutr. 1999; 129: 1765-8.

27 Horner NK, Patterson RE, Neuhouser ML, Mape JW, Beresford SA, Prentice RL. Participant characteristics associated with error in self-reported energy intake from the Women's Health Initiative food frequency questionnaire. Am. J. Clin. Nutr. 2002 [in press].
28 Bingham SA, Cummings JH. Urine nitrogen as an independent validatory measure of dietary intake: a study of nitrogen balance in individuals consuming their normal diet. Am. J. Clin. Nutr. 1985; 42: 1276-89.

29 Kestin M, King I, Yasui Y. Combinations of plasma phospholipid fatty acids as markers of total diet fat intake. FASEB J. 1998; 12: A344. 